\title{
The search for dark matter (contd)
}

\begin{abstract}
An article with an arresting title puts forward a novel way of resolving the question whether the matter that holds the Universe together is 'cold' or 'hot' by showing that one kind of particle might fill both roles.
\end{abstract}

ONE way of making a research article catch the eye is the well-worn trick of uniting in the title a number of disparate concepts, raising in the potential reader's mind the question of how a single article can deal with such a variety of things. Perhaps the best example is the title of the late Jacques Monod's book Chance and Necessity, but here is another, the title of a research article that reads "Bose condensates, Big Bang nucleosynthesis and cosmological decay of a $17 \mathrm{keV}$ neutrino". The author, Jes Madsen from the University of Aarhus, must be sure that hardly any of the readers of Physical Review Letters for 27 July will have failed to pause at page 571 .

What Madsen has to say is as intriguing as the title. The underlying question is whether neutrinos may account for the cosmological dark matter. The $17 \mathrm{keV}$ neutrino is the object for which evidence was provided by J. J. Simpson in 1985 , but which has not yet been generally accepted as one of the menagerie of particles. The $17 \mathrm{keV}$ refers to the supposed mass of the neutrino, which works out at about 3 per cent of the electron mass. So much is clear from the title, but where do "Bose condensation" and "Big Bang nucleosynthesis" fit in?

Bose condensation evidently refers to the behaviour of a collection of bosons (particles with zero or integral spin in the quantum sense). The superfluid component of superfluid helium, for example, is a Bose condensate. What can be the connection with the $17 \mathrm{keV}$ neutrino, which is also supposed to be the neutrino corresponding to the $\tau$-electron? If the neutrino exists, there is some evidence that it must be unstable, and that one of the products of the decay is the more familiar electron neutrino, called $v_{e}$. But in that case, there must be another particle to carry away some of the energy, and because the neutrinos are both fermions (particles with spin half), the missing particle must be a boson.

Indeed, the argument is more general than that. If the $17 \mathrm{keV}$ neutrino is supposed to decay in the simplest possible way into two particles, one must be a fermion (to account for the half-integer spin of the original neutrino), not necessarily the electron neutrino, and the other must be a boson. And there will also be an antineutrino corresponding to the $17 \mathrm{keV}$ particle and then antiparticles corresponding to each of the two decay products. Madsen reminds his readers that the inverse of the decay process will also be possible.

If these $17 \mathrm{keV}$ neutrinos are real, and if they have something to do with dark matter, there must be quite a number of them and, being fermions, their behaviour will be governed by the Pauli exclusion principle (that no more than two may be in the same quantum state), which in turn implies that if the sea of neutrinos is in thermodynamic equilibrium with itself, the energy of the particles (or, more helpfully, their momentum) being distributed according to Fermi-Dirac statistics.

Equilibrium, of course, implies some temperature, which is most conveniently measured in electron-volts (avoiding the use of Boltzmann's constant). Madsen is chiefly concerned with temperatures such those in the early stages of the Big Bang, measured perhaps in MeV. Equilibrium also implies some means of interaction between the particles themselves or with matter of some other kind, supposed to be done through the weak nuclear interaction (and which would have been efficient in the early Big Bang Universe). If the sea of $17 \mathrm{keV}$ neutrinos is indeed a Fermi-Dirac distribution, the number density of neutrinos with a particular momentum will be essentially independent of momentum up to a value comparable with the temperature (in energy units), above which the density will fall off with increasing momentum. (The cut-off would be abrupt if the temperature were zero.)

It is then straightforward (if complicated) to calculate the number-density of the decay products as a function of momentum, after making an assumption about the decay halflife. (The complication is the need to transform from the rest-frame of the particle, in which each decay product will have the same momentum, to the frame of the real Universe.) And this is where the interest lies. Being a fermion and a boson, and given that the weak nuclear interaction should bring them into equilibrium among themselves, the decay products should themselves satisfy Fermi-Dirac and BoseEinstein statistics respectively.

Several unexpected things follow from this, not the least of which is that the effective speed of the decay of the $17 \mathrm{keV}$ neutrinos is constrained by the inverse reaction. Even if the half-life of the $17 \mathrm{keV}$ neutrino in isolation is as little as a tenth of a second, the inverse reaction between the decay products to recreate $17 \mathrm{keV}$ neutrinos will increase the effective half-life to about 100,000 seconds - rather more than a day.

The bosons involved in this process, whatever they may be, raise other possibilities. With momenta distributed by Bose-
Einstein statistics (whose characteristic is that low or even zero-momentum states are occupied preferentially), if the bosons have a small mass, a fraction of them may form a Bose condensate, which means that they will move as if they were a collective whole. Just what fraction of bosons would condense in this way into the lowest energy state depends on the temperature and the lifetime, but Madsen argues that as much as a third of the decay bosons might be thus contained.

That immediately provides a new way of looking at the dark-matter conundrum: if a fraction of the bosons hang together collectively and if each of them has a small amount of mass, they will behave individually as particles of 'cold' dark matter with negligible kinetic energy. But the remainder will be energetic, and will behave as if they were 'hot' dark matter. Cosmology, in the aftermath of the COBE detection of fluctuations and recent surveys revealing more largescale galaxy clustering than expected, may require quantities of both hot and cold dark matter. So one intriguing feature of Madsen's argument is that one kind of particle may account for both the cold and the hot dark matter. All that is left to do, his readers will conclude, is to find that boson.

That accounts for the "Bose condensation" in the title of Madsen's paper. What about the "nucleosynthesis"? In the standard model of the Big Bang, the formation of the light nuclei such as deuterium and ${ }^{4} \mathrm{He}$ is powerfully influenced by weak interactions with particles such as neutrinos, notably electron neutrinos. The point here is that neutrinos should have appeared on the scene before the ratio of protons and neutrons in the Universe was fixed. But if one of the decay products of the $17 \mathrm{keV}$ neutrinos is indeed the electron neutrino, the energy distribution of those particles will be altered and so will be the yield of the different light nuclei.

Madsen is especially pleased that his outline of what the $17 \mathrm{keV}$ neutrino will do for the early Universe is to admit a somewhat lower abundance of primaeval ${ }^{4} \mathrm{He}$ than in the standard calculation. He notes that estimates of this abundance of ${ }^{4} \mathrm{He}$ have been falling steadily over the years and that, although the latest estimates are marginally consistent with the standard calculation, the decay of a $17 \mathrm{keV}$ neutrino would allow a lower helium abundance without affecting calculations of the primordial production of deuterium, ${ }^{3} \mathrm{He}$ and ${ }^{7} \mathrm{Li}$, with which the data are more closely in agreement.John Maddox 